Jane Mitchell, Professor in Pharmacology and Critical Care Medicine at Imperial College School of Medicine, London.

But not all COX2 inhibitors are created equal. Rofecoxib, valdecoxib and other COX2 inhibitors in development are highly selective for COX2. Celecoxib, however, inhibits COX2 to a lesser extent and can also partially inhibit COX1. "At the structural level, we can't really explain the difference in selectivity," says Dan Simmons, Professor of Chemistry and Biochemistry at Brigham Young University, Utah. "We know that valdecoxib, celecoxib and rofecoxib share a three-ring structure, but all differ in the central ring, and very small changes on these rings can make a great difference."

This situation resembles the statins, which have been linked to a serious muscle-wasting disorder called rhabdomyolysis at high dosages. Cerivastatin (Baycol; Bayer), one of the more potent statins, seemed to have a greater tendency to cause rhabdomyolysis and was withdrawn. The loss of a competitor helped to cement atorvastatin (Lipitor; Pfizer) as the biggest-selling drug worldwide. So, could history be repeating itself for Pfizer? Does it now have a better drug because of lower selectivity? Could more selective mean more problems?
Perhaps not, says John Wallace, Professor of Pharmacology and Therapeutics at the University of Calgary, Alberta. "Celecoxib does have some inhibitory activity on COX1, which would reduce its potential cardiovascular activity. But a point that is often missed is that the gastrointestinal safety profile of celecoxib is no better than that of conventional NSAIDs."

Any effect of selectivity is likely to be diluted by the large degree of variability in response between patients on the same drug, says Garret FitzGerald, Professor of Medicine and Pharmacology at the University of Pennsylvania. "Regardless of selectivity for COX2, the clinical substrate will affect the response. If you have a risk of cardiovascular disease, then this will augment the response."

"COX1/COX2 selectivity is but one determinant of this variability," says Carlo Patrono, Director at the MURST Center of Excellence on Aging, Chieti, Italy. "There is substantial overlap in COX2 selectivity between celecoxib, and some traditional NSAIDs, such as nimesulide and diclofenac. "It should be emphasized that despite being widely prescribed, NSAIDs have been investigated inadequately with respect to their cardiovascular safety, a fact that the current controversy on COX2 inhibitor safety fails to take into proper consideration," says Patrono.

FitzGerald says companies need to identify the factors that predispose individuals to cardiovascular risk — something that could be investigated with the patients in the APPROVe study. They also need to provide more data on all the COX2 inhibitors. "If you buy in to the idea that COX2 inhibitors decrease the risk of gastrointestinal bleeding, it looks like you will have to buy into the possibility that COX2 inhibition will increase cardiovascular risk in some people," says FitzGerald. "After the VIGOR trial, the burden of proof rested with researchers to prove the hypothesized risk. Now, the burden of proof has shifted to companies who say that the risk does not extend to their drugs to prove that this is a molecule-specific effect."

Simmons agrees that more data are needed. "The biggest question for me is whether valdecoxib, with its similar selectivity to rofecoxib, has a similar risk of cardiovascular events." This is all the more important because a pro-drug of valdecoxib called parecoxib was turned down by the US FDA after a controlled trial revealed a cluster of cardiovascular events.

Pfizer said that two small clinical trials showed that heart bypass surgery patients who

\title{
Better antibiotics through chemistry
}

\section{Chemical issues are a major challenge in developing new antibiotics.}

\section{Simon Frantz}

A call-to-arms for research into novel antibiotics has reignited the debate concerning how to overcome the barriers to discovering and developing these much-needed treatments.

Business, regulatory and scientific issues all provide disincentives for companies to invest in antibiotic research and development, writes

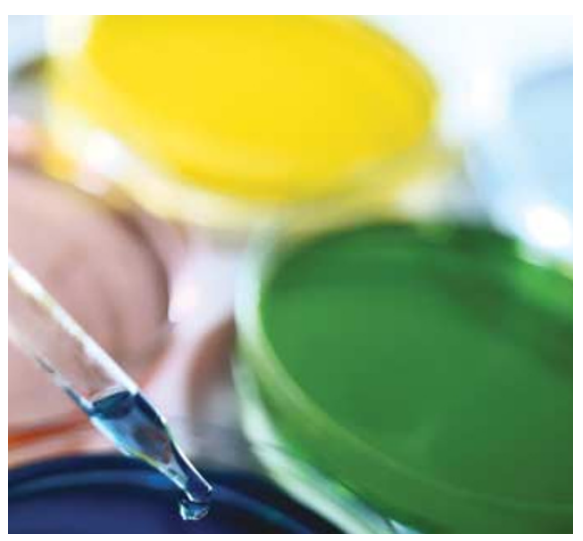

Chemistry issues hinder antibiotic development
Carl Nathan from Cornell University, New York, in a commentary in Nature (Nature 431, 899-902 (2004)). So, a not-for-profit drug company, encouraged by tax incentives, and staffed by industry professionals and executives on sabbaticals, could potentially address this need.

An important issue, which is often overlooked, is the science underlying the development of novel antibiotics, says Christopher Lipinski, Adjunct Senior Research Fellow at Pfizer Global R\&D, and member of the Scientific Advisory Committee for the Global Alliance for Tuberculosis Drug Development.

Alternatives to standard tools and technologies are badly needed. "Combinatorial libraries, so widely used in screening against many noninfectious disease target types, have proved almost totally worthless against infectious disease targets," says Lipinski.

Antibiotics tend to violate Lipinski's 'Rule-of5' guidelines: they are often larger, more complex in chemical structure, more hydrophilic and have a higher charge than small-molecule drugs for non-infectious diseases. Many antibiotics can be injected, so they aren't constrained by the limitations that oral drugs face in order to reach their intended target.

But many chemical libraries contain predominantly Rule-of-5-compliant chemicals, says Stevan Projan, Vice President, Biological Technologies at Wyeth Research. "We need more molecular complexity in the compound libraries themselves," he says.

Traditionally, the major source of antibiotics has been natural products, but for years there has been a decrease in industry investment in this area of research.

Natural-product screening is tedious, and tends to identify many old classes of drugs, says Karen Bush, Biology Team Leader, Antimicrobial Agents Drug Discovery at Johnson \& Johnson. "When Discovery programmes are run with tight deadlines, there is sometimes a reluctance to allow for the time lag between initial identification of activity and eventual isolation of sufficient amounts of material to conduct preclinical safety and efficacy testing."

What's needed are a good natural-products library, especially with novel sources of natural products; analytical chemists to determine novel structures; and medicinal chemists to enhance the activities of natural products to make them act as better drugs, says Projan. "The hard part is finding novel sources and novel natural products," says Projan. "The others are 
took valdecoxib had an increased risk of stroke and heart attack, although re-examination of its clinical database of 8,000 patients with rheumatoid arthritis and osteoarthritis showed no increased cardiovascular risk in those taking valdecoxib for up to a year. Pfizer also said that it will be conducting a large study to see whether celecoxib increases the risk of heart attacks in osteoarthritis patients.

Merck said that its COX2 inhibitor in development, etoricoxib (Arcoxia), showed no significant difference in the number of serious side effects in osteoarthritis patients than those treated with diclofenac.

The European Medicines Agency is reviewing safety data on all COX2 inhibitors, and the FDA will hold an Advisory Committee in early 2005 to discuss the risk. One of the biggest issues they will face is putting the cardiovascular risk into context: does the risk of taking rofecoxib, and potentially other COX2 inhibitors, outweigh the risk of complications

doable but rarely considered up front by most biotechs embarking on drug discovery programmes."

"The major issues hampering the development of novel molecules have been in the identification, synthesis and optimization of active natural product 'hits' identified from various biological extracts," says Peter Tambros, CEO of VivoQuest, a natural-products-based company in Valley Cottage, New York.

Many antibiotics have been made by semisynthetic modifications. Any modification has to not only improve the pharmacokinetics and pharmacodynamics of a drug, but, in the case of antibiotics, it must also try to overcome the problem of antibiotic resistance. As natural products are generally more structurally complex than small-molecule drugs, extensive modifications are tricky and complete synthesis of natural-productlike compounds is challenging.

However, minor modifications of novel natural compounds by semisynthetic methods shouldn't present too much of a problem, says Christopher Walsh, Professor at the from gastrointestinal bleeding from taking NSAIDs and aspirin?

"You can't compare apples and oranges," says James Fries, Professor of Medicine at the Stanford University School of Medicine. "For most people the heart attack risk is pretty scary and the frequencies are similar in magnitude. More importantly, there are many alternatives, old and new, that are essentially as GI safe and do not increase cardiovascular risk."

"Selective inhibitors of COX2 remain a rational choice for patients at low cardiovascular risk who are at high risk of serious gastrointestinal complications, especially while taking traditional NSAIDs," says FitzGerald. "It would also seem prudent to avoid COX2 inhibitors in patients who have or are at risk of cardiovascular disease."

Patrono agrees, adding that "caution should apply to all inhibitors of COX2 because they would have the same impact on prostacyclin biosynthesis in the face of suppressed thromboxane biosynthesis."

Department of Biological Chemistry and Molecular Pharmacology at Harvard Medical School. Usually, chemists pick one or two sites for modification, says Walsh. "From this point of view, many — probably most - of the natural products that are therapeutic candidates will offer one or a few sites for selective modification."

So, there is the science to turn this crisis around, says Solomon Nwaka, Scientific Officer at the Medicines for Malaria Venture. "With appropriate resources, a clear structural strategy and support from all sectors, this impending gap in availability of antibiotics can be prevented."

Pooling these talents can only be beneficial, says Nathan. "What we have now is a great deal of expertise in antibiotic development including first-hand knowledge, earned at great cost, of what does not work - that is being scattered as teams are broken up," he says. "We need a venue where teams can come back together with a charge to develop antibiotics, and the tools and compounds suited to the task."

\section{NEWS IN BRIEF}

\section{Good news for malaria vaccine...}

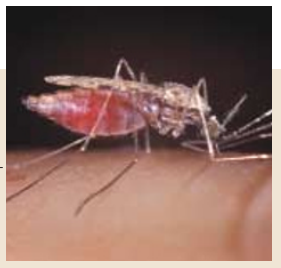

The Phase llb success of a malaria vaccine developed by GlaxoSmithKline and the Malaria Vaccine Initiative (MVI) has provided the first real hope for protection against the disease. RTS,S/ASO2A acts against the most deadly strain of the malaria parasite, Plasmodium falciparum, at the 'pre-erythrocytic stage'; that is, before the red blood cells become infected. During the first six months of the trial in Mozambique on 2,000 healthy children aged $1-4$ years, $58 \%$ fewer children developed severe malaria with the vaccine, which is a fusion of immunogenic components from the surface of $P$. falciparum sporozoites with hepatitis B surface antigen, plus a proprietary adjuvant. Alonso, P. et al. Lancet 364, 1411-1420 (2004).

\section{...but bad news for flu vaccine}

Chiron has announced that it will not supply its influenza vaccine Fluvirin for the 2004-2005 influenza season, due to bacterial contamination of some of the doses. The United States felt the brunt of this vaccine shortage, as Chiron was expected to supply nearly half of the 100 million doses expected. Aventis-Pasteur, the other supplier of flu vaccines in the US, said it had made some extra doses of its flu vaccine to help address the problem.

\section{Parkinson's trial halted}

One of the most promising treatments in development for Parkinson's disease has faced a setback. Initial analysis of the preliminary Phase II data showed no clinical improvement in patients given Amgen's glialderived neurotrophic factor (GDNF) compared with placebo after six months of treatment. But 4 out of the 34 subjects produced antibodies to the GDNF protein, raising fears that this could trigger a dangerous immune reaction with prolonged treatment. Researchers are trying to understand the discrepancy between these results and an earlier trial on 5 patients with GDNF who showed a dramatic recovery in their movements.

\section{Nobel Prizes announced}

The Nobel Prize in Chemistry for 2004 was jointly awarded to Aaron Ciechanover, Avram Hershko and Irwin Rose for the discovery of ubiquitin-mediated protein degradation. Their work showed how a molecule called ubiquitin acts as a 'kiss of death' by fastening onto a protein to be destroyed, and accompanying it to the proteasome, where it is degraded. The Prize in Physiology or Medicine went to Richard Axel and Linda Buck for their discoveries of odour receptors and the organization of the olfactory system.

\section{Dogs sniff out cancer}

Man's best friend could now be even more precious, as their supersensitive noses might be able to detect cancer. Carolyn Willis at Amersham Hospital, UK, and her team trained six dogs of varying breeds to identify the urine of patients with bladder cancer, as it is thought that tumours release molecules into the urine that have a characteristic smell. The trained dogs were asked to choose between laboratory dishes of seven types of urine and to lie down in front of the one from a cancer patient. The dogs were correct more than $40 \%$ of the time, much greater than the $14 \%$ figure that

would be expected if they chose by chance.

Willis C. M. et al. BMJ 329 ,

712-714 (2004).

For more news and analysis go to news@nature.com www.nature.com/news 